\title{
PUBLIC MENTAL HEALTH IN POST-COVID-19 ERA
}

\author{
Fei-Fei Ren ${ }^{1} \&$ Rong-Juan Guo ${ }^{2}$ \\ ${ }^{I}$ Beijing University of Chinese Medicine, Beijing, China \\ ${ }^{2}$ Department of Neurology, Dongfang Hospital, Beijing University of Chinese Medicine, Beijing, China
}

received: 29.5.2020;

revised: 9.6.2020;

accepted: 16.6 .2020

\section{SUMMARY}

Transmission of the 2019 novel coronavirus (COVID-19) has now rapidly spread around the world, which has alarming implications for individuals and communities, in particular for public mental health. Significant progress has been made in the prevention and control of the COVID-19 pandemic in China, but the psychological crisis caused by the epidemic is still not over and may continue to exist. The public mental health in the post-COVID-19 era should not be ignored. This article provides early warning for the public's mental health in the post-COVID-19 era by listing the characteristics and duration of the public mental health crisis following the SARS outbreak. In addition, based on the current situation, specific methods and measures are proposed in order to provide effective reference for the prevention and control of psychological crisis caused by the COVID-19 epidemic.

Key words: COVID-19 - post-COVID-19 era - psychological crisis - mental health - prevention and control

\section{INTRODUCTION}

At the end of 2019, the Coronavirus disease 2019 (COVID-19) caused by severe acute respiratory syndrome coronavirus-2 (SARS-CoV-2) broke out rapidly and widely spread allover the world (Wang et al. 2020). As of 17:00 on May 25, 2020, Beijing time, there were 5,304,772 cases confirmed COVID-19 and 342,029 deaths in more than 200 countries and regions around the world according to the daily COVID-19 epidemic report announced by World Health Organization (WHO 2020). In most cases, emerging infectious diseases (EIDs) always been a major burden on the global economy and public health (Jones et al. 2008), the COVID-19 epidemic has also posed a serious threat to global public health security and economy (Bedford et al. 2020). What's more, the rapid transmission and mortality risk of the COVID-19 infection may increase the risk of mental health problems among healthy individuals in the general public.

As a major public health emergency, people is under great physical and psychological pressure from COVID19 (Xiang et al. 2020). This epidemic has brought not only the risk of death but also the psychological pressure that hard to erase, such as excessive stress, insomnia, anxiety, depression and so on (Kang et al. 2020). Since the outbreak of this epidemic, both China and foreign scholars have carried out research on the psychological health problems of different vulnerable populations caused by COVID-19. With the improvement of the epidemic situation in China, the resumption of work and production across the country is being carried out in an orderly manner, but the psychological crisis brought by the epidemic is still not over, while ciral transmission, incubation times, and care of severely affected patients rightly dominate the news. Therefore, it is still necessary to correctly assess various collateral damage in this post- epidemic period, also called "post-COVID-19 era", especially mental health problems (Vervoort et al. 2020). Here, this paper is to explore the psychological crisis that the public still has and may continue in the postCOVID-19 era, and to propose relevant countermeasures for reference.

\section{PUBLIC PSYCHOLOGICAL CRISIS DURING COVID-19 EPIDEMIC}

More than half of the interviewees have mental health problems in major public health emergencies and have a strong need for psychological assistance (Wainberg et al. 2017). Of course, the COVID-19 is no exception. As a sudden stressor, the outbreak of this disease not only has a serious impact on the normal life of the public, but also has a great impact on mental health. This situation could be caused by many factors, like a large number of viral infections diffusion, the anxious from ordinary people, strict public health measures that restriction of personal freedoms, the growing economic pressure, the intensive work of health care workers, the continuously delayed start of school and work and even the confusing news of the epidemic on the internet. All of these are great sources of stressors that will promote the widespread emotional distress unquestionably, such as anxiety, depression, confusion, panic, insecurity and even sadness among different groups of people. Also, it will increased the danger for mental illness associated with COVID-19. Fortunately, Extensive research in public mental health associated with the COVID-19 outbreak has established with different vulnerable populations, which include infected patients, medical staff, students, and the general population, revealed that emotional distress is ubiquitous among affected populations. A study investigated 1,210 ordinary people from 194 cities in China with their psychological status at the 
early stage of COVID-19 epidemic and found that $53.8 \%$ of the respondents had mild to moderate symptoms of anxiety and depressive, and about $16.5 \%$ of the respondents had moderate to severe symptoms of depressive, while $28.8 \%$ had moderate to severe anxiety (Wang et al. 2020). At the same time, research on the psychological state of medical staff members has also been reported. A survey on the psychological states of 1,257 medical staff members in Wuhan, China, showed that the prevalence of depression, anxiety, insomnia and pessimism caused by the COVID-19 outbreak was $50.4 \%, 44.6 \%, 34.0 \%$ and $71.5 \%$, respectively (Lai et al. 2020). Another study investigated the mental health status of 7,143 college students from Changzhi medical college, China during the COVID-19 outbreak by using cluster sampling, indicated that about $0.9 \%$ of the respondents had severe anxiety symptoms, $2.7 \%$ of the population moderate anxiety, and about $21.3 \%$ percent had mild anxiety (Cao et al. 2020). The author ends with a strong appeal that more attention should be monitored to the mental health of college students during epidemics.

Not only that, similar studies have been reported both in domestic of China and abroad, involving different vulnerable populations. From these studies, it can be seen that all kinds of people are under great mental and psychological stress and challenges during the COVID-19 outbreak, and the long-term outcomes of the mental health interventions need further evaluation to determine how we can ameliorate the negative effects of this viral outbreaks in the general public, especially among the elderly, children, pregnant women, disabled people, patients with serious chronic diseases and other key groups as well as vulnerable groups.

\section{PSYCHOLOGICAL PROBLEMS IN THE POST-COVID-19 ERA STILL NEEDS ATTENTION}

At present, important strategic achievements had been made in the response to the COVID-19 epidemic in China. According to the data from the official website of the National Health Commission of China, as of 24:00 on May 25, 2020, Beijing time, according to reports from 31 provinces (autonomous regions and municipalities directly under the central government) and Xinjiang production and construction corps, there were currently 81 confirmed cases (including 7 severe cases) and 5 suspected cases (National Health Commission of China 2020). At present, the domestic epidemic of China has been controlled effectively, while the explosive growth of the COVID-19 outside China has continued. The focus of China's epidemic prevention and control has been shifted from "internal non-proliferation and external export" to "external import and internal rebound prevention", and the work stalled due to this infectious diseases is gradually resuming (National Health Commission of China 2020). However, at the same time, it is still necessary to be aware that the various mental and psychological problems caused by the COVID-19 epidemic may not be over.

This concern can be amply illustrated by the impact of the severe acute respiratory syndrome (SARS) epidemic. As an acute respiratory infectious disease caused by SRAS coronavirus (SARS-CoV), SRAS broke out in Guangdong, China, and rapidly spread to southeast Asia and the world in 2003, causing a global epidemic wave like the COVID-19 outbreak (Yang et al. 2020). Until the mid-2003, the SARS epidemic was gradually eliminated, however, its psychological impact on the public was far from over. A study investigated the mental complications for survivors after 30 months of SARS outbreak through a cohort study, the result showed that the cumulative incidence of mental disorders even up to $58.9 \%$, about a quarter of the survivors combined the chronic post-traumatic stress disorder (PTSD), and the prevalence of depression disorder related to the SARS outbreak was $15.6 \%$. Also, the multivariate analysis confirmed that the women and chronic diseases before the SARS were independent predictors of PTSD (Mak et al. 2009, 2010). Another study focused on the psychological stress of the survivors one year after the SARS outbreak, showing that the survivors still under elevated stress and psychological distress. The General Health Questionnaire (GHQ-12) from survivors scored an alarming proportion (64\%), which suggests the psychiatric morbidity. At the same time, this study showed a worrying trend that during the SARS outbreak, health care workers experienced similar levels of psychological stress to non-health care workers, but this changed significantly one year after the outbreak, more obvious psychological stress levels, posttraumatic symptoms, anxiety, depression symptoms and higher GHQ-12 scores occurred among health care workers (Lee et al. 2007). Mcalonan's research showed that health care workers who were on the frontline of SARS not only have obvious chronic stress but also higher levels of anxiety and depression (Mcalonan et al. 2007). In addition, A long-time study which continuously followed up the mental and fatigue conditions from 233 SARS survivors in Hong Kong showed that at an average of 41.3 months after the SARS epidemic, more than $40 \%$ of the respondents had frequent mental and psychological symptoms and about $40.3 \%$ had chronic fatigue problems (Lam et al. 2009).

The above research fully demonstrates that the mental and psychological problems caused by excessive stress in the epidemic not only exist during the epidemic, but also be concerned in the post-epidemic era. What is clear now is that both the COVID-19 pathogen named SARS-CoV-2 and the SARS-CoV are belong to the $\beta$ coronavirus genus, and with $79 \%$ similarity in gene sequence, and both of them infect cells through angiotensin converting enzyme 2 (ACE2). Nonetheless, the transmissibility of SARS-CoV-2 is stronger than that of SARS-CoV, and the number of deaths caused by the COVID-19 and the affected scope are also significantly higher than that of SARS (Walls et al. 2020, Rabaan et 
al. 2020). So, It can be speculated that the mental and psychological problems caused by COVID-19 to the public will continue to exist in the post-COVID-19 era and may become a trigger for many other diseases. Therefore, in view of the current special stage, we should continue to pay attention to the long-term consequence of the COVID-19 pandemic for our mental health, so as to provide a basis for early intervention.

\section{SUGGESTIONS ON PREVENTION AND CONTROL OF PSYCHOLOGICAL CRISIS IN POST-COVID-19 ERA}

\section{Carry out prospective cohort studies actively}

Cohort study is an observational research method that divide a specific population into two groups exposed to a certain factor and not exposed to a certain factor or several subgroups with different exposure levels, track their respective outcomes, and compare the incidence of an outcome in two groups or some groups, and determine whether there is a causal association between the exposure factor and the outcome and the degree of association (Grimes et al. 2002). Prospective cohort study is the basic form of cohort study. At the beginning of the study, the observed objects did not reach the outcome, but knew their exposure properties, and then compared the outcomes of different groups after follow-up a certain period of time. So it is also known as real-time cohort study or longitudinal study. Now, the prospective cohort study has been widely used in various chronic diseases research, aging-related problems and the relationship between environmental exposure and diseases (Euser et al. 2009).

As with all crises, it is essential that we monitor innovations and collect high-quality date from COVID19 so that, at a later date, we can consider what worked and what did not. Affected by the development of the epidemic situation, most of the current researches on COVID-19 and public psychological crisis are crosssectional investigations, and the conclusions only shows the correlation between them. However, if we want to know whether the epidemic is a risk factor of psychological crisis or other diseases for the public, a longitudinal cohort study is needed. That is to say, based on the known risk factors of psychological crisis, the high-quality date related to COVID-19 and psychological crisis are maintained as much as possible, and more reliable research data are used to formulate evidence-based prevention and treatment strategies, so as to reduce the adverse psychological impact caused by COVID-19 pandemic.

\section{Formulate guidelines for post-COVID-19 era mental rehabilitation}

According to the data released by the National Health Commission of China, a total of 78,219 COVID19 cases have been cured and discharged so far. Strengthening the follow-up and health management of discharged patients is an important part of disease prevention and rehabilitation. At present, a number of rehabilitation protocols and guidelines have been issued for COVID-19 recovery in China. For example, in view of respiratory impairment and physical dysfunction caused by COVID-19, the Chinese Association Of Rehabilitation Medicine (CARM) has issued a respiratory rehabilitation guidance after COVID-19, which provides clinical guidance and basis for patients' respiratory rehabilitation (Chinese Association Of Rehabilitation Medicine 2020). The guidelines on the reasonable selection and standard application of Traditional Chinese medicine (TCM) rehabilitation techniques in the treatment of COVID-19 been published (Li et al. 2020). Meanwhile, a protocol for integrated traditional Chinese and western medicine rehabilitation has also been developed to guide the late recovery of COVID-19 patients (Xia et al. 2020). On the whole, the psychological rehabilitation advices of COVID-19 are mentioned in the above guidance or consensus, but the specific rehabilitation measures and methods are rarely discussed. Given the widespread and persistent nature of the psychological crisis caused by COVID-19, it is necessary to develop specific post-epidemic psychological rehabilitation norms, including specific and feasible rehabilitation suggestions and operational methods, in order to promote public mental and psychological health in the post-COVID-19 era.

\section{Building a public psychological crisis management platform in the Post-COVID-19 era based on "Internet +".}

With the development of the information age, Internet medical care has profoundly changed people's cognitive of health and the mode of seeking medical care (Loh et al. 2016). In the early stage of the COVID-19 pandemic, "Internet + ", with its unique connectivity features and convenient, cross-regional and non-contact means, became a technological breakthrough for China's medical service system to cope with the epidemic. Through the "Internet + " model, governments at all levels and academic groups have provided a large number of psychological counseling to the public through Internet hospitals, cloud care and other means to help the COVID-19 prevention and control (Liu et al. 2020). In the post-COVID-19 era, continue to build a service platform for public psychological crisis management based on "Internet +" technology, especially included in primary medical institutions or create an "Internet + medical" model. An online psychological diagnosis and treatment and counseling platform centered on community health service centers has been established to provide timely psychological health services to the public. At the same time, the medical supervision function of the Internet can also be used to regular the follow-up of COVID-19 discharged patients, in order to timely detection of problems, early intervention. 


\section{Improve the ability of community medical staff to identify and manage psychological crisis after the COVID-19}

With the spread of the COVID-19, communities have become the front line of epidemic prevention and control. Community health service institution is an important carrier of community prevention and control, grassroots medical staff have been the most direct responders. During the COVID-19 pandemic, grass-roots medical staff played an important role in pre-screening and triage, door-to-door visits, follow-up and site screening. However, grass-roots communities are still the "main battlefield" for public mental health in the postCOVID-19 era. Therefore, education and training on psychosocial issues should be provided to community health service professionals at the grass-roots level. Help them to identify and manage the psychological crisis after COVID-19 epidemic, and understand the common forms of psychological crisis, master the measures and methods to deal with psychological crisis, and actively communicate with the public to understand their psychological state, and realize the early detection and prevention of psychological crisis. Also, the evidence-based resources related to mental health with COVID-19 should be established and widely distributed. In addition, the occurrence of mental disorders of healthcare workers after a major epidemic was negatively correlated with the training and support they received in the early stage (Lancee et al. 2008). Therefore, improving the psychological crisis management ability for grass-roots medical staff can also provide a mental health guarantee for themselves.

\section{CONCLUSIONS}

Mental health is an important cornerstone of health (Prince et al. 2007). Currently, mental and psychological diseases may rank first in the global burden of disease, on the same level as cardiovascular diseases and circulatory diseases (Vigo et al. 2016). It is also become one of the three major factors affecting the disability survival years (YLDs) in the burden of disease in China (Zhou et al. 2019). Therefore, the psychological crisis caused by COVID-19 pandemic should not be ignored. The health challenges of the post-COVID19 era have been noted. For example, some scholars worry that the emergence of a large number of chronic cardiovascular disease in the postCOVID-19 era will lead to a short-term re-strain of medical resources or a surge in the work pressure of medical staff (Allahwala et al. 2020). Naser Moqhadasi A also proposed that neurologists will face great difficulties in the treatment of multiple sclerosis in the post-COVID-19 era (Naser Moqhadasi 2020). Taking history as a mirror, the persistent psychological crisis of the public after the SARS pandemic has sounded the alarm for the public's mental and psychological health in the post-COVID-19 era.
In the future, we still need to pay attention to the persistent psychological crisis caused by the COVID-19 pandemic, and explore the rules at the scientific research level through longitudinal cohort study. By formulating psychological rehabilitation norms in the post-COVID-19 era, building a public psychological crisis management platform based on "Internet +", and improving the ability of grassroots medical staff to identify and manage psychological crisis, the public's mental health in the post-epidemic era can be guaranteed.

\section{Acknowledgements: None.}

Conflict of interest: None to declare.

\section{Contribution of individual authors:}

Fei-Fei Ren: literature searches, writing manuscript, approved the final version.

Rong-Juan Guo: concept and design of this article, literature searches, read and approved the final version.

\section{References}

1. Allahwala UK, Denniss AR, Zaman $S \&$ Bhindi $R$ : Cardiovascular disease in the post-COVID-19 era - the impending tsunami? Heart Lung Circ 2020; S14439506(20)30129-3

2. Bedford J, Enria D, Giesecke J, Heymann DL, Ihekweazu $C$, Kobinger $G$ \& WHO Strategic and Technical Advisory Group for Infectious Hazards: COVID-19: towards controlling of a pandemic. The Lancet 2020; 395:1015-1018

3. Cao W, Fang Z, Hou G, Han M, Xu X, Dong J \& Zheng J: The psychological impact of the COVID-19 epidemic on college students in China. Psychiatry Res 2020; 287:112934

4. Chinese Association Of Rehabilitation Medicine: Guidelines on respiratory rehabilitation for covid-19 (second edition). Zhonghua Jie He He Hu Xi Za Zhi 2020; 308-314 [in Chinese]

5. Euser AM, Zoccali C, Jager KJ \& Dekker FW: Cohort studies: prospective versus retrospective. Nephron Clin Pract 2009; 113:c214-c217

6. Grimes DA \& Schulz KF: Cohort studies: marching towards outcomes. The Lancet 2002; 359:341-345

7. Jones KE, Patel NG, Levy MA, Storeygard A, Balk D, Gittleman JL \& Daszak P: Global trends in emerging infectious diseases. Nature 2008; 451:990-993

8. Kang L, Li Y, Hu S, Chen M, Yang C, Yang BX \& Liu Z: The mental health of medical workers in Wuhan, China dealing with the 2019 novel coronavirus. Lancet Psychiatry 2020; 7:e14

9. Lai J, Ma S, Wang Y, Cai Z, Hu J, Wei $N \&$ Hu S: Factors associated with mental health outcomes among health care workers exposed to coronavirus disease 2019. JAMA Netw Open 2020; 3:e203976

10. Lam MH, Wing YK, Yu MW, Leung CM, Ma RC, Kong AP \& Lam SP: Mental morbidities and chronic fatigue in severe acute respiratory syndrome survivors: long-term follow-up. Arch Intern Med 2009; 169:2142-2147 
11. Lancee WJ, Maunder RG, Goldbloom DS \& Coauthors for the Impact of SARS Study: Prevalence of psychiatric disorders among Toronto hospital workers one to two years after the SARS outbreak. Psychiatr Serv 2008; 59:91-95

12. Lee AM, Wong JG, McAlonan GM, Cheung V, Cheung C, Sham PC \& Chua SE: Stress and psychological distress among SARS survivors 1 year after the outbreak. Can J Psychiatry 2007; 52:233-240

13. Li JS \& Zhang HL: Expert consensus on rehabilitation of Chinese medicine for COVID-19(First Edition). Acta Chinese Medicine 2020; 35:681-688 [in Chinese]

14. Liu S, Yang L, Zhang C, Xiang YT, Liu Z, Hu S \& Zhang $B$ : Online mental health services in China during the COVID-19 outbreak. Lancet Psychiatry 2020; 7:e17-e18

15. Loh KK \& Kanai R: How has the internet reshaped human cognition? Neuroscientist. 2016; 22:506-520

16. Mak IW, Chu CM, Pan PC, Yiu MG \& Chan VL: Longterm psychiatric morbidities among SARS survivors. Gen Hosp Psychiatry 2009; 31:318-326

17. Mak IW, Chu CM, Pan PC, Yiu MG, Ho SC \& Chan VL: Risk factors for chronic post-traumatic stress disorder (PTSD) in SARS survivors. Gen Hosp Psychiatry 2010; 32:590-598

18. McAlonan GM, Lee AM, Cheung V, Cheung C, Tsang KW, Sham PC \& Wong JG: Immediate and sustained psychological impact of an emerging infectious disease outbreak on health care workers. Can J Psychiatry 2007; 52:241-247

19. Naser Moghadasi A: The big challenge for neurologists in treating patients with multiple sclerosis in the postCOVID-19 era. Mult Scler Relat Disord 2020; 42:102170

20. National Health Commission of China: Interpretation of the circular on further strengthening the work of infection prevention and control in medical institutions in order to implement the requirements of regular epidemic prevention and control (in Chinese). 2020. Available on: http://www.nhc.gov.cn/yzygj/s3594/202005/4f8326122836 421c9d50bfla074402ee.shtml

21. National Health Commission of China: Update on COVID-19 as of 24:00 on 25 May (in Chinese). 2020. Available on: http://www.nhc.gov.cn/xcs/yqfkdt/202005/ 02e547cdbb654065bf523b61e03f3ddb.shtml

22. Prince M, Patel V, Saxena S, Maj M, Maselko J, Phillips MR \& Rahman A: No health without mental health. The Lancet 2007; 370:859-877

23. Rabaan AA, Al-Ahmed SH, Haque S, Sah R, Tiwari R, Malik YS \& Rodriguez-Morales AJ: SARS-CoV-2, SARS-
CoV, and MERS-COV: a comparative overview. Infez Med 2020; 28:174-184

24. Vervoort D, Luc JGY, Percy E, Hirji S \& Lee R: Assessing the collateral damage of the novel coronavirus: a call to action for the post-COVID-19 era. Ann Thorac Surg 2020; S0003-4975(20)30618-4

25. Vigo D, Thornicroft $G \&$ Atun R: Estimating the true global burden of mental illness. Lancet Psychiatry. 2016; 3:171-178

26. Wainberg ML, Scorza P, Shultz JM, Helpman L, Mootz JJ, Johnson KA \& Arbuckle MR: Challenges and opportunities in global mental health: a research-to-practice Perspective. Curr Psychiatry Rep 2017; 19:28

27. Walls AC, Park YJ, Tortorici MA, Wall A, McGuire AT \& Veesler D: Structure, function, and antigenicity of the SARS-CoV-2 spike glycoprotein. Cell 2020; 181:281292.e6

28. Wang C, Horby PW, Hayden FG \& Gao GF: A novel coronavirus outbreak of global health concern. The Lancet 2020; 395:470-473

29. Wang C, Pan R, Wan X, Tan Y, Xu L, Ho CS \& Ho RC: Immediate psychological responses and associated factors during the initial stage of the 2019 coronavirus disease (COVID-19) epidemic among the general population in China. Int J Environ Res Public Health 2020; 17:1729

30. World Health Organization: Coronavirus disease (COVID-19) situation report-126. 2020. Available on: https://www.who.int/docs/default-

source/coronaviruse/situation-reports/20200525-covid19-sitrep-126.pdf?sfvrsn $=887 d b d 66 \_2$

31. Xia WG, Hua $Q$, Wang G, Guo TC, Zhu SS, Zhao $Y$ \& Zhang BL: Standard for rehabilitation diagnosis and treatment of COVID-19 with integration of traditional Chinese and Western medicine. Rehabilitation Medicine 2020; 30:85-92 [in Chinese]

32. Xiang YT, Yang Y, Li W, Zhang L, Zhang $Q$, Cheung $T$ \& $\mathrm{Ng} \mathrm{CH}$ : Timely mental health care for the 2019 novel coronavirus outbreak is urgently needed. Lancet Psychiatry 2020; 7:228-229

33. Yang Y, Peng F, Wang R, Guan K, Jiang T, Xu G \& Chang $C$ : The deadly coronaviruses: the 2003 SARS pandemic and the 2020 novel coronavirus epidemic in China. J Autoimmun 2020; 109:102434

34. Zhou M, Wang H, Zeng X, Yin P, Zhu J, Chen W \& Liang $X$ : Mortality, morbidity, and risk factors in China and its provinces, 1990-2017: a systematic analysis for the Global Burden of Disease Study 2017. The Lancet 2019; 394:1145-1158

Correspondence:

Rong-Juan Guo, MD

Department of Neurology, Dongfang Hospital, Beijing University of Chinese Medicine

1 Chenzhuang street, Changxindian, Fengtai district, Beijing 100029, China

E-mail: 381160563@qq.com 